\title{
Large-Eddy-Simulation-based analysis of complex flow structures within the volute of a vaneless centrifugal pump
}

\author{
TAIMOOR ASIM* ${ }^{*}$ and RAKESH MISHRA \\ School of Computing and Engineering, University of Huddersfield, Huddersfield HD1 3DH, UK \\ e-mail: t.asim@hud.ac.uk; r.mishra@hud.ac.uk
}

MS received 2 May 2016; revised 15 November 2016; accepted 7 December 2016

\begin{abstract}
Centrifugal pumps are very common in many fluid handling industrial applications, such as petrochemicals, oil and gas, etc. Although the design practices for centrifugal pumps are well established, efforts are directed towards optimising such systems for better operational efficiencies. In order to optimally design centrifugal pumps, it is beneficial to first understand the complex flow phenomena within different sections of the pump for a variety of operating conditions. This is normally achieved through the use of modern techniques, such as Computational Fluid Dynamics (CFD), where the flow within centrifugal pumps can be numerically modelled and important flow features can be analysed for better understanding of interactions amongst different process variables. CFD offers different turbulence modelling techniques with an aim to predict realistic flow approximations. Large Eddy Simulation (LES) offers a more accurate solution to this, in which the larger eddies are resolved while smaller eddies are modelled; hence predictions using LES are more realistic. Further, in turbulence modelling within centrifugal pumps, it is also important to model the complete interaction amongst different variables rather than a simplistic single blade passage flow analysis. In the present work, the complex blade-tongue interactions and their consequent effects on the pressure fluctuations within the volute have been evaluated. It is seen that the secondary flow features in the near-tongue regions due to blade interactions with the tongue affect the flow characteristics within the volute considerably.
\end{abstract}

Keywords. Large Eddy Simulation; centrifugal pump; sliding mesh; vorticity; blade-tongue interaction.

\section{Introduction}

Centrifugal pumps are widely used in many engineering fluid handling applications. The design and performance prediction of centrifugal pumps involve systematic investigation of a number of interdependent parameters. Computational Fluid Dynamics (CFD)-based approaches are hence widely used for performance prediction of centrifugal pumps alongside experimental investigations. CFDbased analyses are employed for the hydrodynamic design of various pump types as numerical simulations offer reasonably accurate information on the fluid behaviour within various components of the system, assisting the engineers to obtain performance envelope of a particular design [1-3].

Numerical investigations to enumerate the effect of the rotational speed on the velocity vectors in a single blade passage of a centrifugal pump were carried out by Hussein and Hasan [4]. The standard $k-\varepsilon$ turbulence model was employed in order to analyse the flow structures within the pump. Detailed investigations into the velocity vectors revealed that the flow velocity is generally lower on the

*For correspondence suction side, which progressively increases up to the leading edge of the blades. Numerical investigations regarding the complex flow phenomena within a centrifugal pump using Unsteady Reynolds Averaged Navier-Stokes (URANS) approach were carried out by Liu et al [5]. Turbulence was modelled using Shear Stress Transport $k-\omega$ model, and the results predicted at shut-off conditions of the pump were validated against Particle Image Velocimetry (PIV) measurements. It was reported that two eddies existed within each impeller blade passage and the velocity at the volute exit was very low. Furthermore, it has been reported that the pressure fluctuations that dominate the volute region are of the same order as that of blade passing frequency.

Several researchers investigated the flow features within the impeller region of a centrifugal pump using both numerical and experimental techniques [6, 7]. RANS, URANS and Large Eddy Simulation (LES) were employed to predict the flow features within a pump turbine. It has been reported that both the RANS and URANS models were not able to predict the flow behaviour accurately, while the LES-based results matched well with the PIV data. It has been shown that URANS underestimates performance characteristics and overestimates pressure 
fluctuations. Similarly, it has been observed that LES predicts energy-discharge characteristic curve more accurately than RANS, and that the torque-predicting capability of LES is superior to that of RANS. These works were extended further to investigate the complex flow structure within the diffuser of a mixed-flow nuclear reactor coolant pump [8]. The authors have reported that the flow structures within diffuser channels are closely associated with the position of the diffuser channel relative to the casing nozzle, and LES has been employed to critically analyse this.

Unsteady complex flow phenomena within the volute of a centrifugal pump have been investigated numerically in detail by Zhang et al [9]. The authors used LES-based approach and have reported that the distinct peaks observed in the pressure build-up curve of the pump are closely associated with the positioning of the impeller blades. Unsteady vortical structures in the near-tongue region are greatly influenced by the relative orientation of the impeller blades with respect to the tongue, and the upstream effects of the tongue significantly affect the vorticity distribution on the blade pressure side. Furthermore, it has been stated that the rotor-stator interaction is dominated by the vortex shedding in the wake of the blade trailing edge and vortical structure's impingement on the tongue with subsequent cutting and distortion.

Harmonic Balance Method (HBM) was used by Magagnato et al [10] in order to predict the complex unsteady flow features within the impeller passages of a centrifugal pump. The results obtained have been verified against data collected from PIV. The production and eventual dissipation of the vortical structures within the impeller passages have been reported in detail. Furthermore, it has been reported by Yao et al [11] that hybrid RANS-LES model performs the best in predicting the flow features within a centrifugal pump, and can clearly capture the vortical structures near the impeller exit and near tongue regions. Researchers used LES-based approach in order to predict the pressure fluctuations within the volute region of the centrifugal pump, and extended it to predict the hydrodynamic noise generation [12,13]. Distinct pressure peaks have been reported in both these studies and it has been reported that the Sound Pressure Level (SPL) increases with the flow rate, and that the highest SPL is predicted in the tongue region.

Investigations on pressure fluctuations within the volute of centrifugal pumps have been reported by many researchers; however, spatio-temporal variations of pressure within the different sections of the volute have not been investigated in detail. Hence, in the present study, LES-based approach has been used to numerically investigate the complex flow features within a centrifugal pump, focusing on the volute and tongue regions. Furthermore, the spatio-temporal history of the pressure (and head) fluctuations within different sections of the volute has been reported in detail.

\section{Numerical modelling of the centrifugal pump}

Unlike most recent numerical studies concerning the centrifugal pump's analysis based on LES, where normally only one section of the impeller is modelled, this study analyses the complete centrifugal pump model. The centrifugal pump that has been numerically modelled is model FH32/200AH from Perdrollo. This specific pump has five backward-type impeller blades, where the impeller diameter is $215 \mathrm{~mm}$. The inlet and outlet of pump have a diameter of 50 and $32 \mathrm{~mm}$, respectively. The radial distance between the volute and the tongue is $4.5 \mathrm{~mm}$. The construction of the pump complies with the standards EN 733 and DIN 24255 [14]. Figure 1 depicts the numerical model of the pump under consideration. An entrance length of $1.56 \mathrm{~m}$ has been specified in the present study, based on the calculated Reynolds number at the inlet of the flow domain (Eq. (1)), in order to let the flow become fully developed before entering the impeller eye [15]:

$$
\frac{L_{e}}{D}=4.4 R e^{\frac{1}{6}}
$$

where $L_{e}$ is the equivalent length (entrance length) and $D$ is the inlet pipe's diameter. Furthermore, an outlet pipe of $1.5 \mathrm{~m}$ has been attached to the outlet of the pump. This has been purposefully done as per the literature so that the boundary conditions should be far away from the region of interest [16]. Furthermore, it has been analysed and confirmed that the outlet boundary is at such a distance from the outlet of the pump that the disturbances from within the pump do not interfere with the outlet boundary.

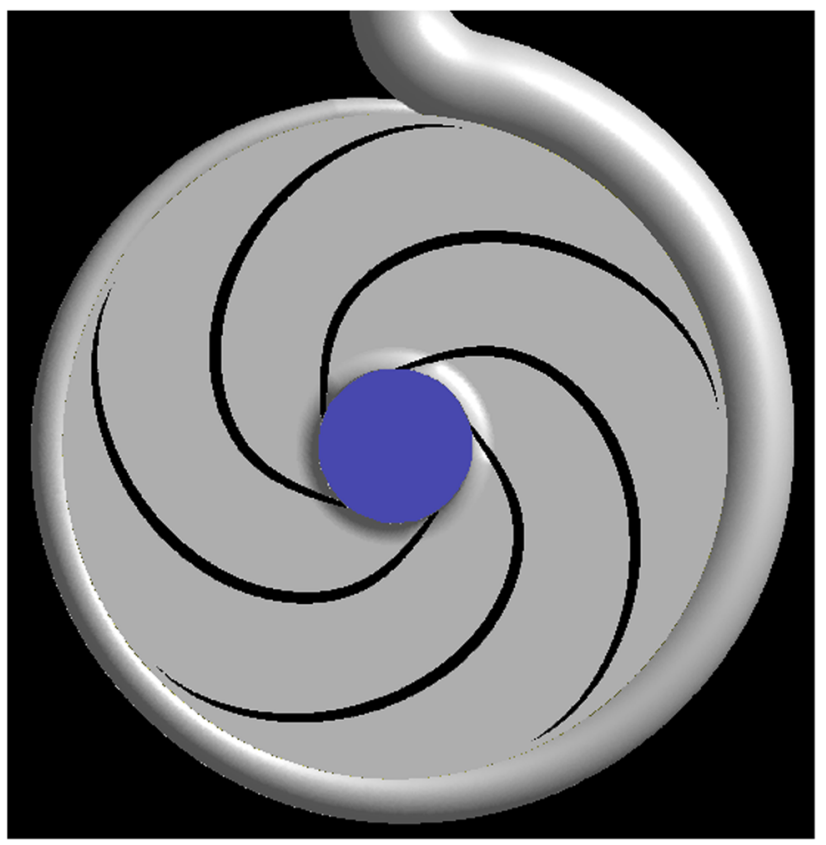

Figure 1. Numerical model of the pump. 
LES has been used in the present study. LES is a numerical technique to solve transient turbulent flows. In this technique, the large flow scales are resolved while the smaller scales are numerically modelled. The large flow scales are more energetic, anisotropic in nature and are mostly dependent on the fluid, flow and geometric variables. The smaller flow scales are assumed to be isotropic and universal [17]. In order to differentiate between the larger and smaller flow scales, filtering operation is often employed. The filtered mass and momentum conservation equations for incompressible flow of fluid are

$$
\begin{gathered}
\frac{\partial \bar{u}_{i}}{\partial x_{i}}=0 \\
\frac{\partial \bar{u}_{i}}{\partial t}+\frac{\partial \bar{u}_{i} \bar{u}_{j}}{\partial x_{j}}=-\frac{1}{\rho} \frac{\partial \bar{P}}{\partial x_{i}}+\frac{\partial}{\partial x_{j}}\left(v \frac{\partial \bar{u}_{i} \bar{u}_{j}}{\partial x_{j}}\right)-\frac{\partial \tau_{i j}^{s g s}}{\partial x_{j}} .
\end{gathered}
$$

A second-order implicit scheme is used for temporal discretisation. Semi-Implicit Method for Pressure Linked Equations (SIMPLE) algorithm is employed for pressurevelocity coupling. In the SIMPLE algorithm, an approximation of the velocity field is obtained by solving the momentum equation [18]. The pressure gradient term is calculated using the pressure distribution from the previous iteration or an initial guess. The pressure equation is formulated and solved in order to obtain the new pressure distribution. Velocities are corrected and a new set of conservative fluxes is calculated.

The adequacy of a grid resolution for LES is generally determined in terms of the grid spacing in wall units. Considering the complexity of the impeller, an unstructured tetrahedral meshing scheme is employed with about 8 million cells as shown in figure 2 .

Based on the wall shear stresses acting on the different flow domain walls, it has been ensured that the first couple of cells are within the viscous sub-layer region, while another five cells are within the log-law regime. The details of the different $y+$ values and the corresponding cell heights, used in the present study, are summarised in table 1. It can be seen that nine inflation layers have been used in the near-wall region of the flow domain, with the first layer height of $0.009164 \mathrm{~mm}$ and a growth rate of 2 .

The density of the working fluid, i.e. water, is considered to be constant $\left(\rho=998.2 \mathrm{~kg} / \mathrm{m}^{3}\right)$, with dynamic viscosity $(\mu)$ of $0.001003 \mathrm{~Pa}-\mathrm{s}$. Furthermore, the boundary conditions at the inlet and outlet of the flow domain are specified as mass flow inlet of 6, 9, $12 \mathrm{~m}^{3} / \mathrm{h}$ (Best Efficiency Point) and $15 \mathrm{~m}^{3} / \mathrm{h}$, and outflow, respectively. The centrifugal pump under consideration has a horizontal-to-vertical inlet and outlet; hence, care has been taken in order to accurately model this.

Sliding Mesh technique has been used in the present study in order to rotate the impeller blades. The computational domain is divided into rotating and non-rotating

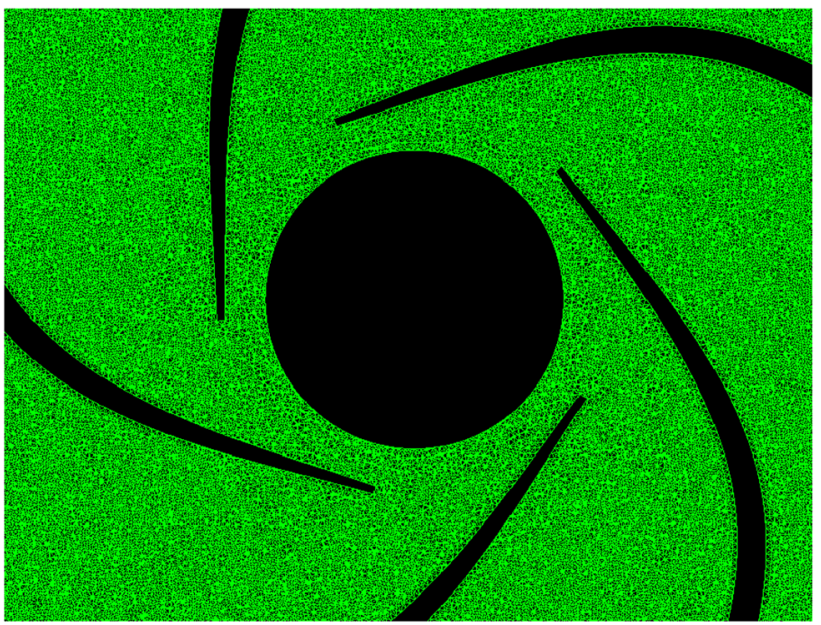

(a)

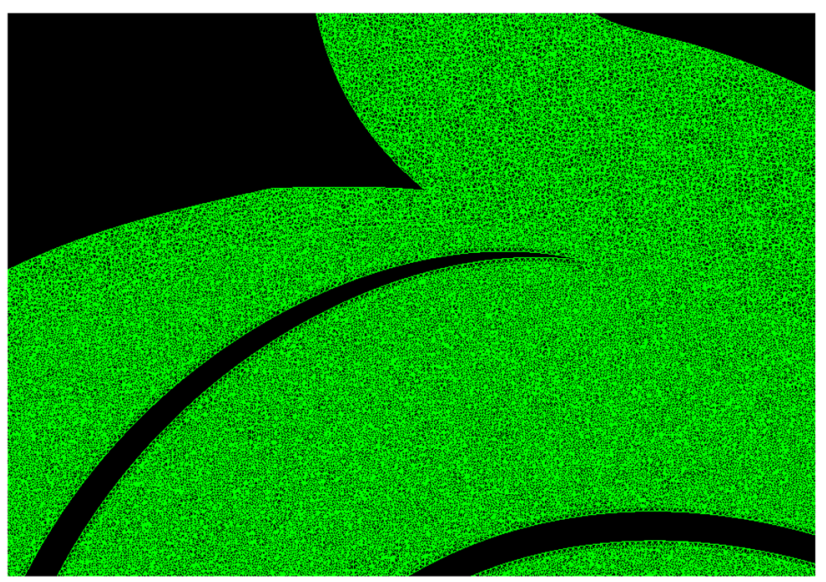

(b)

Figure 2. Mesh in (a) impeller and (b) near-tongue region.

Table 1. Details of $y+$ values.

\begin{tabular}{llc}
\hline$y+$ & $y(\mathrm{~mm})$ & Region \\
\hline 1 & 0.009164 & Viscous sub-layer \\
2 & 0.018329 & Viscous sub-layer \\
4 & 0.036657 & Viscous sub-layer \\
8 & 0.073315 & Buffer layer \\
16 & 0.14663 & Log-law region \\
32 & 0.293259 & Log-law region \\
64 & 0.586518 & Log-law region \\
128 & 1.173036 & Log-law region \\
256 & 2.346073 & Log-law region \\
\hline
\end{tabular}

zones, joined through mesh interfaces. The impeller blades and the attached hub surfaces are treated as a rotating zone at the operating speed. The governing equations in each sub-domain are written with respect to that sub-domain's reference frame. The solver used in the present study enforces the continuity of the absolute velocity to provide 


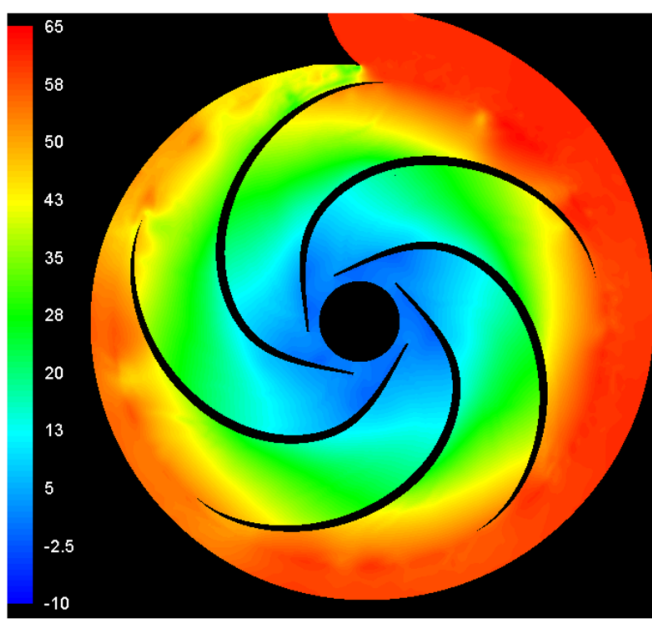

(a)

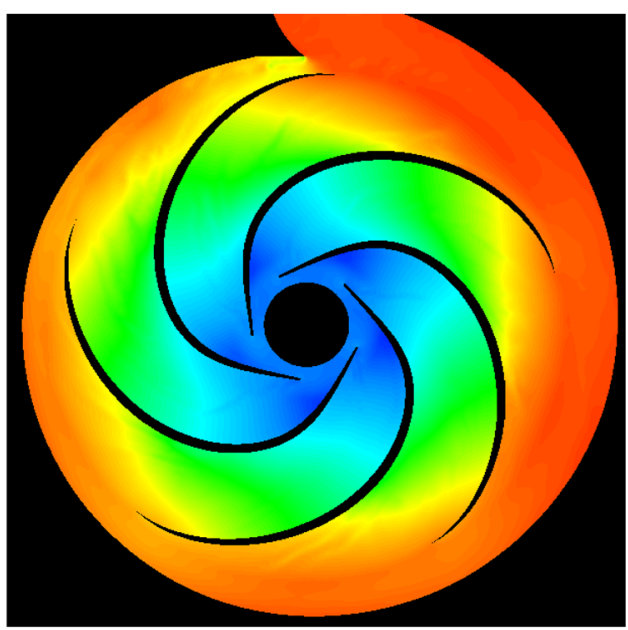

(c)

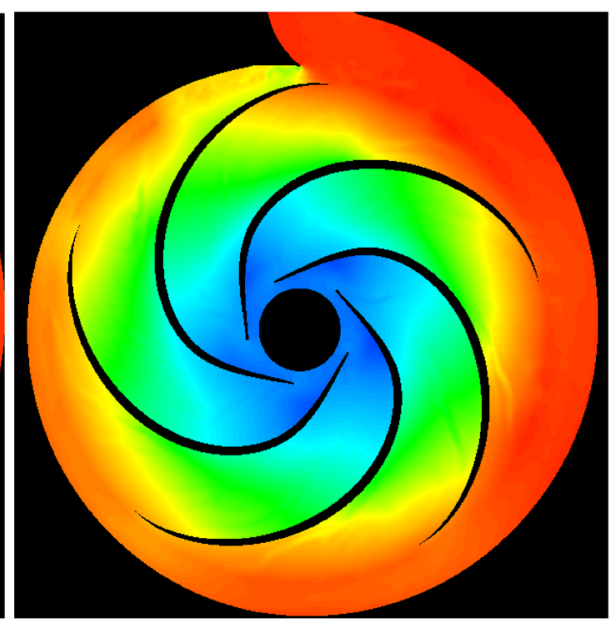

(b)

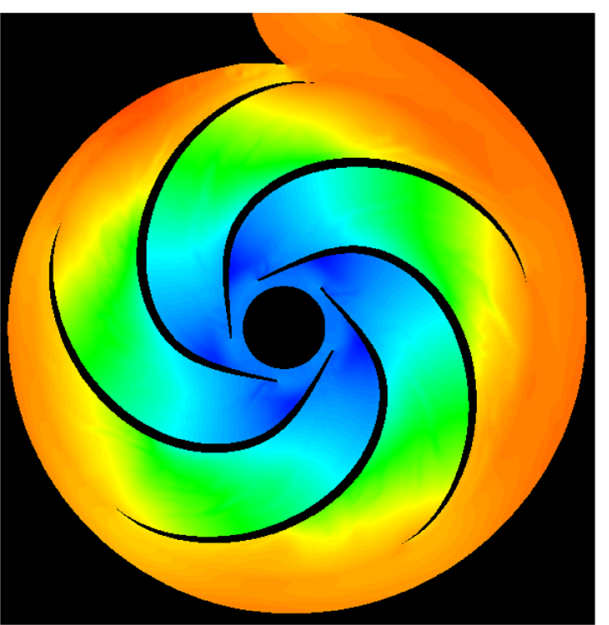

(d)

Figure 3. Variations in the head developed (m) by the pump at (a) $6 \mathrm{~m}^{3} / \mathrm{h}$, (b) $9 \mathrm{~m}^{3} / \mathrm{h},(\mathbf{c}) 12 \mathrm{~m}^{3} / \mathrm{h}$ and $(\mathbf{d}) 15 \mathrm{~m}^{3} / \mathrm{h}$.

the correct neighbouring values of velocity for the subdomain under consideration. When the relative velocity formulation is used, velocities in each sub-domain are computed relative to the motion of the sub-domain. No-slip condition has been specified on the walls of the flow domain. Furthermore, it has been assumed that the walls of the flow domain are hydrodynamically smooth.

Temporal discretisation is very important in case of transient flow analysis. In order to get realistic flow features prediction, a time step size of $5.74713 \times 10^{-5} \mathrm{~s}$ has been specified in the present study, which corresponds to $1^{\circ}$ rotation of the impeller blades. A smaller time step size could also be considered for time-step-independent predictions. It has been observed that this time step size predicts the complex flow structures within the centrifugal pump with reasonable accuracy, which is discussed in the next section.

\section{Results and discussion}

The first step towards realistic evaluation of the flow field within a centrifugal pump is to analyse the important flow parameters such as head developed by the pump, flow velocity, etc. Figures 3 and 4 depict the variations the head developed by the pump, and the flow velocity within the pump, respectively. Figure $3 a-d$ presents the head characteristics at the flow rates of $6,9,12 \mathrm{~m}^{3} / \mathrm{h}$ (BEP) and $15 \mathrm{~m}^{3} /$ $\mathrm{h}$. The scale of the head variations, as well as flow velocity variations, has been kept the same for the different operating conditions of the pump for accurate comparison purposes. It can be seen in figure $3 a-d$ that the head at the impeller eye is negative, which increases along the flow path towards the volute. The maximum head, at any crosssection, is observed only in the volute region, as expected, and the head gradually develops within the volute, i.e., 


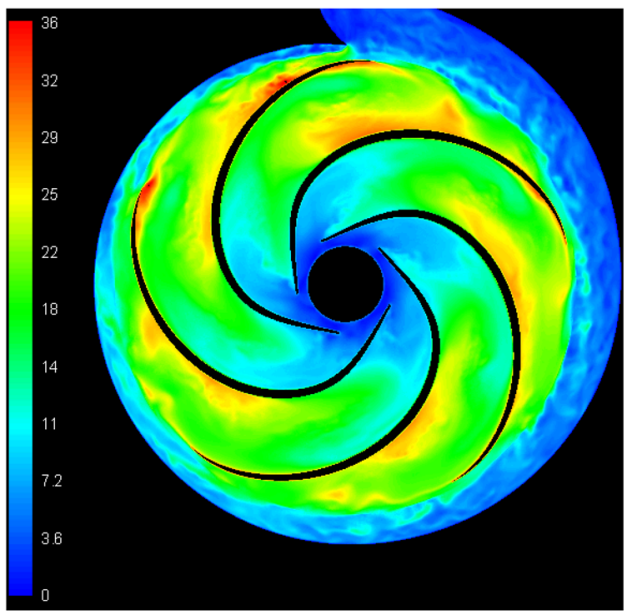

(a)

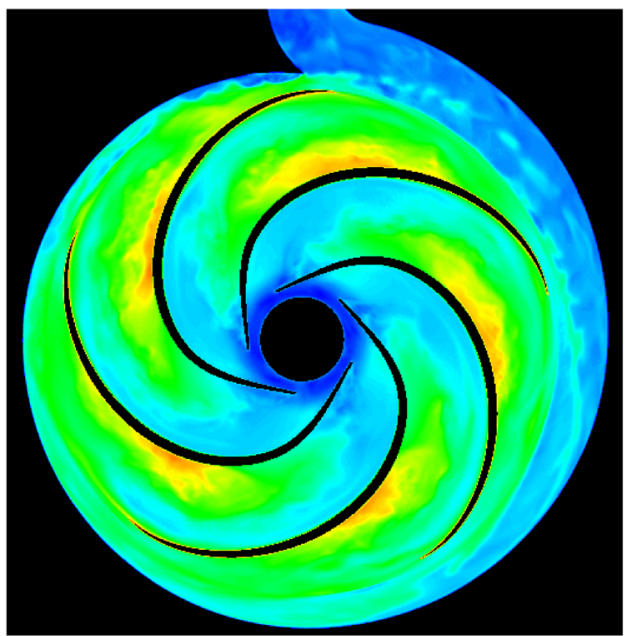

(c)

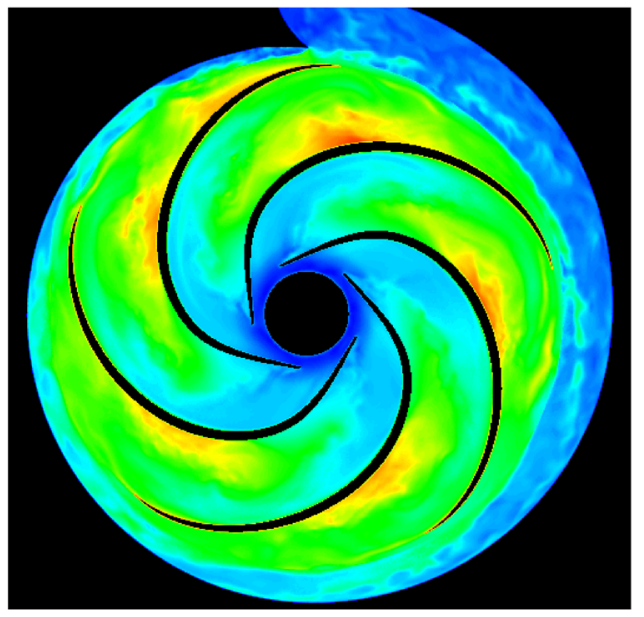

(b)

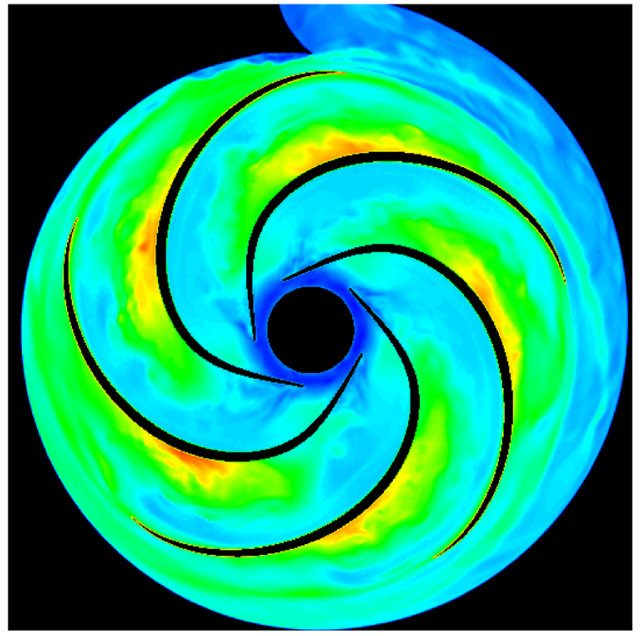

(d)

Figure 4. Variations in the flow velocity (m/s) within the pump at (a) $6 \mathrm{~m}^{3} / \mathrm{h}$, (b) $9 \mathrm{~m}^{3} / \mathrm{h},(\mathbf{c}) 12 \mathrm{~m}^{3} / \mathrm{h}$ and $(\mathbf{d}) 15 \mathrm{~m}^{3} / \mathrm{h}$.

from near-tongue region towards the outlet, in anti-clockwise direction. Hence, the maximum head is observed at the outlet of the pump. This trend is common at each operating condition of the centrifugal pump.

There are however critical variations within the head developed by the pump at various operating conditions. The first, and most obvious, variation noticed is that as the flow rate increases, the head developed by the pump decreases. Moreover, non-uniformities are observed downstream of the tongue region, which are different at different operating conditions of the pump. At $6 \mathrm{~m}^{3} / \mathrm{h}$, which is a near-stall operating point of the pump, these variations are quite significant. As the flow rate increases, these non-uniformities reduce considerably, and it can be seen that at $15 \mathrm{~m}^{3} /$ $\mathrm{h}$, they are restricted to a very small distance downstream of the tongue. Further analysis needs to be carried out to better understand the characteristics of these non-uniformities.
Figure 4a-d depicts that the flow velocity is higher in the impeller region, as compared with the impeller eye and the volute, as expected. Non-uniformities downstream of the tongue region are quite evident in all the figures; however, it can also be seen that these non-uniformities are significantly reduced as flow rate increases; especially, in case of $15 \mathrm{~m}^{3} / \mathrm{h}$ flow rate, these non-uniformities can be observed only in a small distance downstream of the tongue region. This discussion suggests that due to the interaction of the impeller blades with the tongue region, secondary flows are produced, which propagate in the volute, downstream of the tongue region. These secondary flows tend to reduce as flow rate increases.

At this point, it becomes important to analyse the secondary flows in more detail. There are several methods for the detection of secondary flow structures, also known as vortical structures. A method that is based on the velocity 


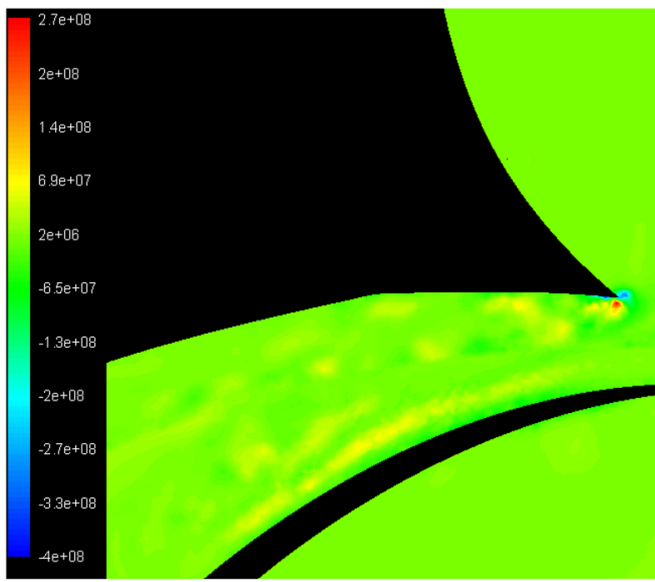

(a)

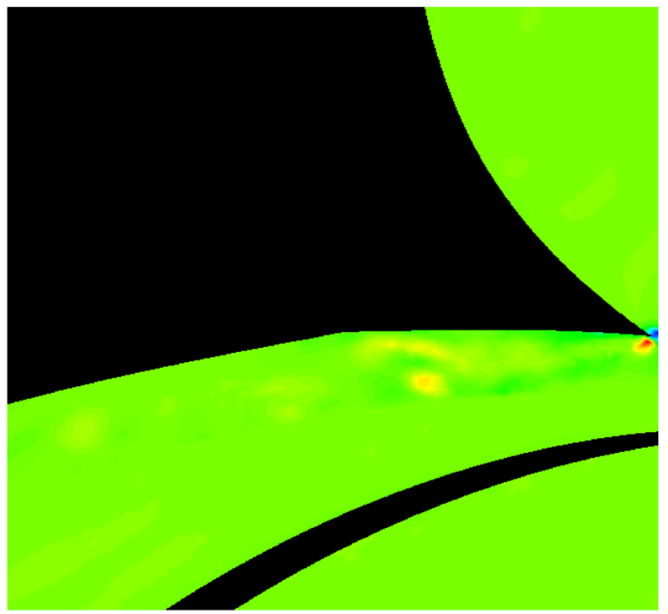

(c)

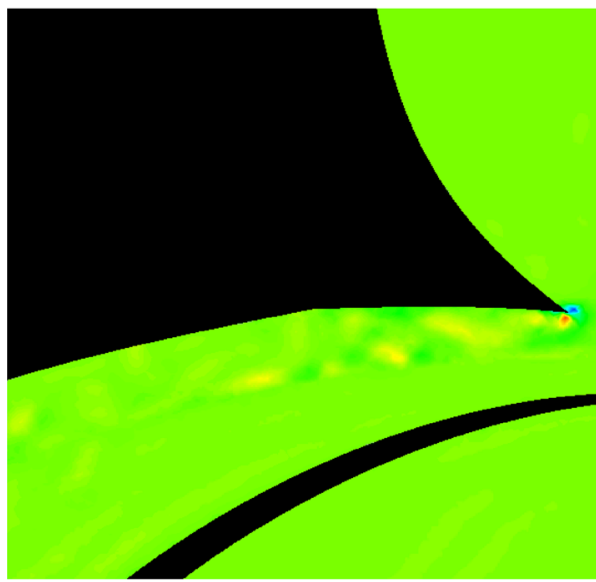

(b)

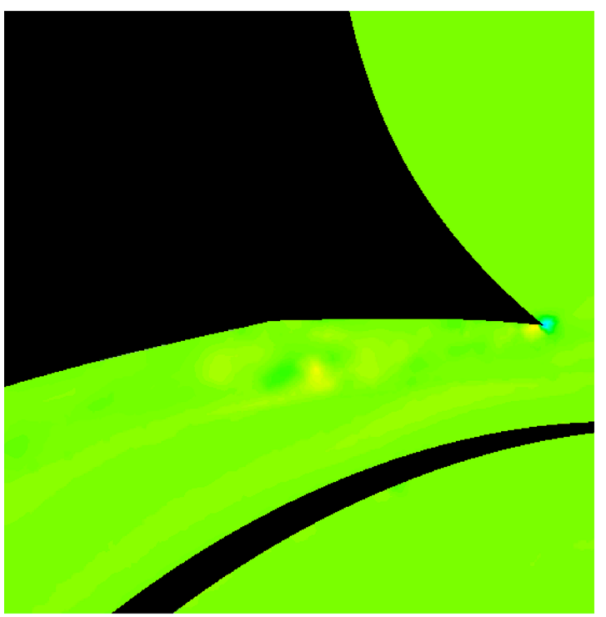

(d)

Figure 5. Variations in $Q$-criterion $\left(/ \mathrm{s}^{2}\right)$ downstream of the tongue region at (a) $6 \mathrm{~m}^{3} / \mathrm{h},(\mathbf{b}) 9 \mathrm{~m}^{3} / \mathrm{h},(\mathbf{c}) 12 \mathrm{~m}^{3} / \mathrm{h}$ and $(\mathbf{d}) 15 \mathrm{~m}^{3} / \mathrm{h}$.

gradient tensor has been adopted in the present study. This criterion, known as the $Q$-criterion, can be expressed as follows:

$$
Q=\frac{1}{2}\left(\|\omega\|^{2}-\|\mathrm{SR}\|^{2}\right)
$$

where $\omega$ is the vorticity (/s) and SR is the strain rate (/s). The $Q$-criterion defines a vortical structure as a connected fluid region with a positive second invariant of the velocity gradient tensor, i.e., $Q>0 / \mathrm{s}^{2}$. This criterion also adds a secondary condition on the pressure, requiring it to be lower than the surrounding pressure in the vortical structure $[19,20]$.

Figure 5a-d depicts the variations in the $Q$-criterion downstream of the tongue region for the various operating conditions of the centrifugal pump considered in the present study. The positive $Q$-criterion values correspond to higher vorticity magnitude than the shear strain rate and vice versa. It can be seen in all the figures that vortical structures are formed at the tongue, and then propagate downstream, in the volute of the pump. Their strength decreases as the distance from the tongue increases. It can be noticed that as the flow rate increases, the strength, and the effective flow area covered by these vortical structures, reduces significantly. At the near-stall operating point, these vortical structures tend to occupy the impeller of the pump as well, and are distributed in the volute. However, as the flow rate increases, these vortical structures become more compact in nature (consuming less effective flow area), and are restricted to the volute region only. These vortical structures lead to increased losses within the centrifugal pump.

In order to quantitatively analyse the performance of the centrifugal pump, figure 6 depicts variations in the instantaneous head developed by the pump for one complete revolution of the impeller blades, at various operating conditions considered in the present study. The dotted lines 


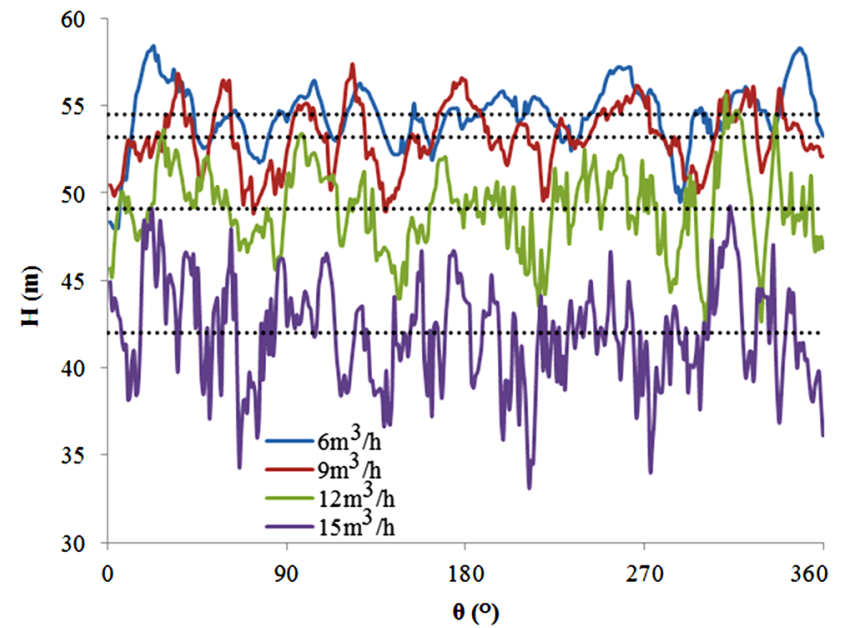

Figure 6. Variations in the instantaneous head developed by the pump at various operating conditions. represent the revolution averages of the instantaneous head curves. It can be seen that as the flow rate increases, both the instantaneous and revolution average heads developed by the pump decrease. The revolution average heads developed by the pump over flow rate change from 6 to $15 \mathrm{~m}^{3} / \mathrm{h}$ are 54.5 to $42 \mathrm{~m}$, respectively. Experimentally measured head values for the same operational range are 55, 52, 48 and $44 \mathrm{~m}$. The differences in the experimentally found head developed by the pump, and predicted revolution average head are $0.9,2.3,2.3$ and $4.6 \%$, correspondingly. This shows that the LES-predicted head values are in close agreement with the experimentally evaluated head.

Apart from verifying LES-predicted head values, figure 6 shows that the instantaneous head characteristics of the centrifugal pump are non-uniform in nature, at all operating points. After careful examination of figure 6 , it was found out that the peaks occur when an impeller blade

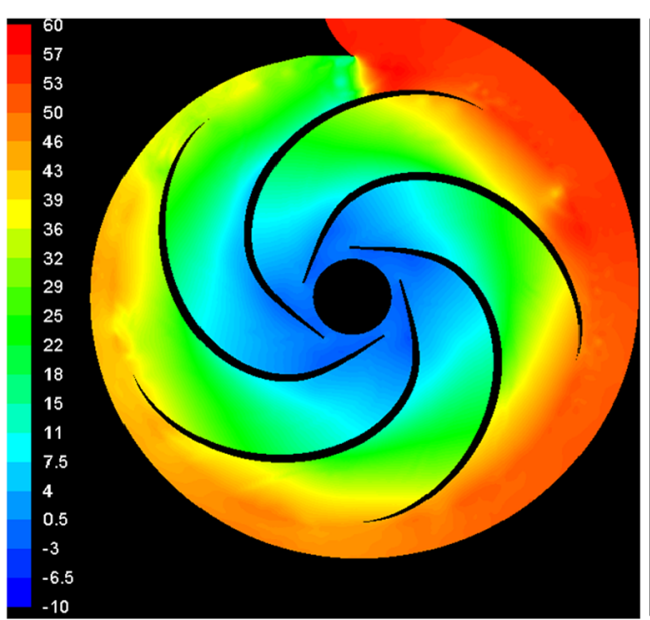

(a)

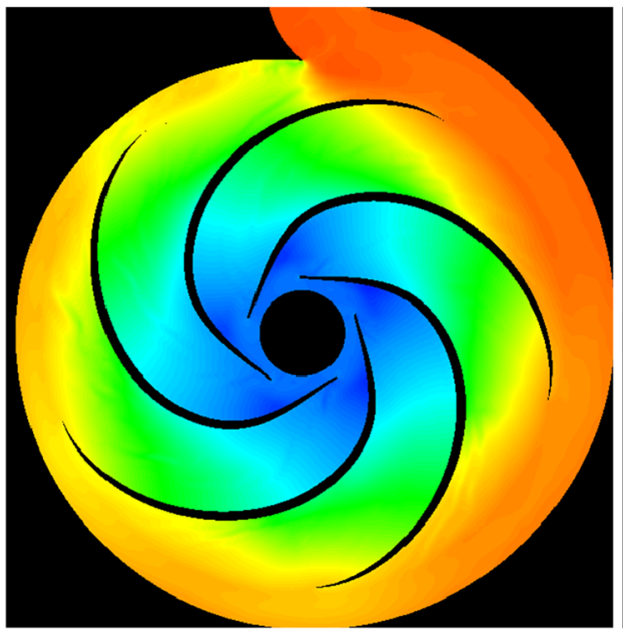

(c)

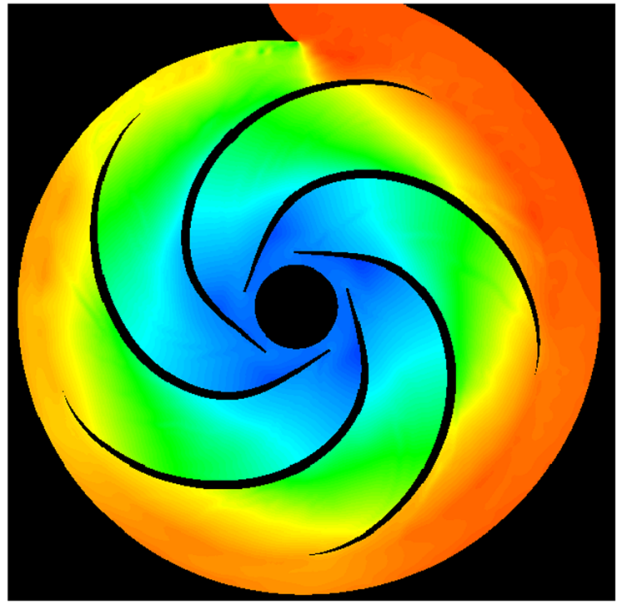

(b)

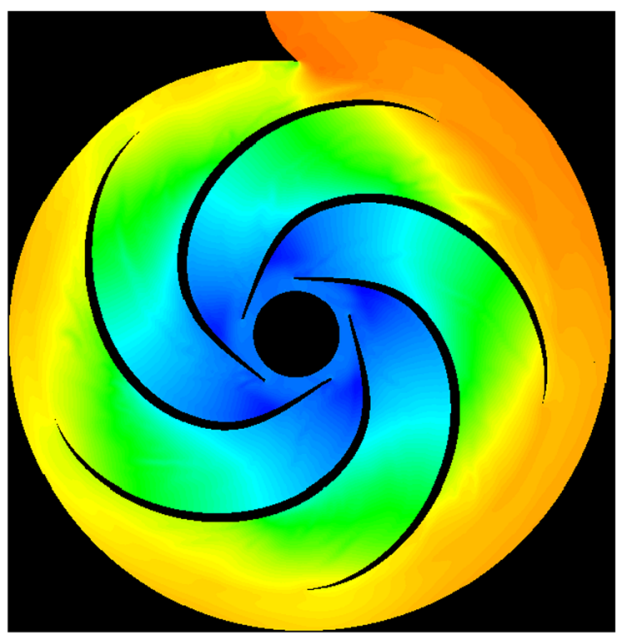

(d)

Figure 7. Variations in the head developed (m) by the pump for lower head configuration at (a) $6 \mathrm{~m}^{3} / \mathrm{h},(\mathbf{b}) 9 \mathrm{~m}^{3} / \mathrm{h},(\mathbf{c}) 12 \mathrm{~m}^{3} / \mathrm{h}$ and (d) $15 \mathrm{~m}^{3} / \mathrm{h}$. 
is in-line with the tongue, while the lower head points occur when the tongue is in-between two impeller blades. The flow parameters associated with the higher heads have already been discussed. The flow parameters associated with the lower heads of the centrifugal pump are discussed hereafter, with special attention to the $Q$-criterion that indicates the losses in the near-tongue region. It is expected that these losses will be significantly higher for lower head configurations.

Figure $7 \mathrm{a}-\mathrm{d}$ depicts head variations within the centrifugal pump at different flow rates. The scale of these figures is from -10 to $60 \mathrm{~m}$, whereas it was -10 to $65 \mathrm{~m}$ in case of higher head configurations, indicating lower head developed by the centrifugal pump. Apart from the scale of the head variations, the rest of the flow structure remains almost the same, i.e., high head developed in the volute, and the maximum head observed at the outlet of the pump. Moreover, secondary flows are dominant in case of lower flow rates, which reduce in both strength and size as the flow rate increases.

Similarly, figure 8a-d depicts flow velocity variations within the centrifugal pump for lower head configuration, at different flow rates. The scale in this case has increased to 0-39 m/s, as compared with $0-36 \mathrm{~m} / \mathrm{s}$ in case of higher head configuration. One obvious difference in the flow structure for lower head configuration, as compared with higher head one, is that as the flow rate increases, the reduction in secondary flows is not as significant. From the comparison of figures $8 \mathrm{~d}$ and $4 \mathrm{~d}$, it is evident that the secondary flows extend further downstream of the tongue region in case of lower head configuration. One possible explanation of this is the geometrical orientations of the impeller blades themselves. In case of lower head configuration, the tongue region is in-between two impeller blades. Hence, the flow propagating towards the outlet of the pump tends to enter into the volute through the wide

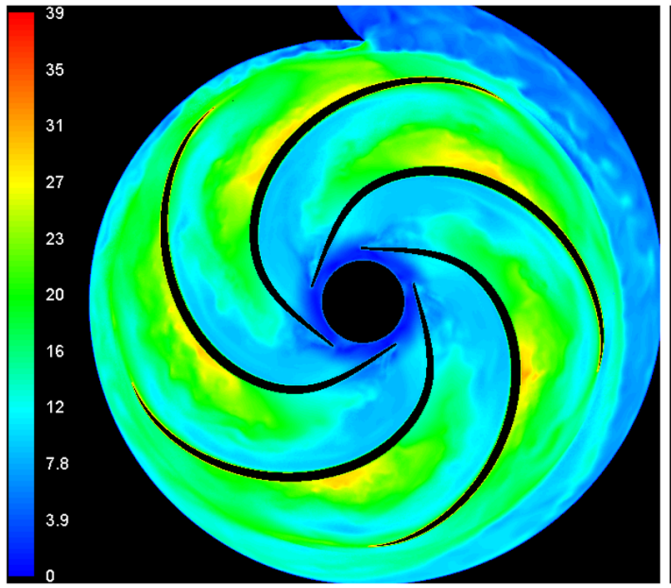

(a)

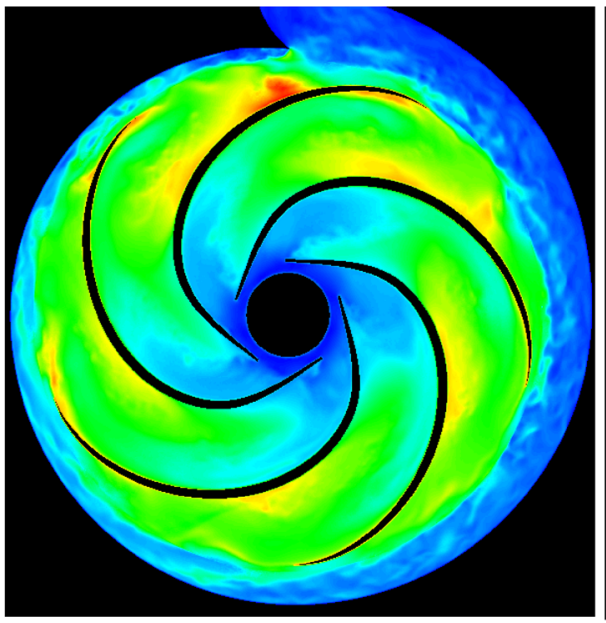

(c)

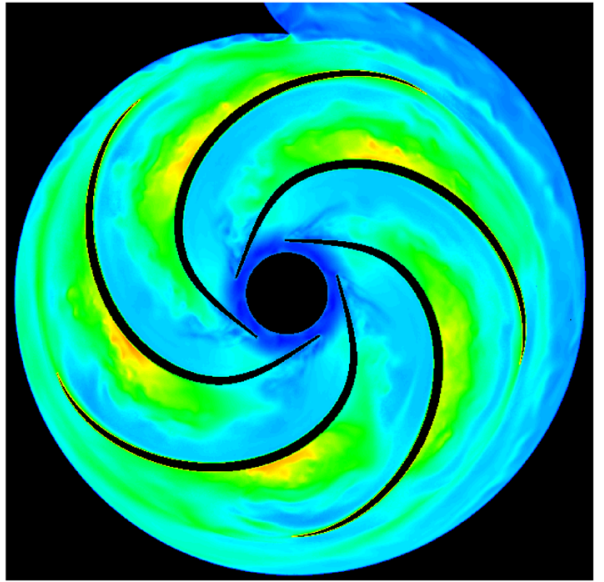

(b)

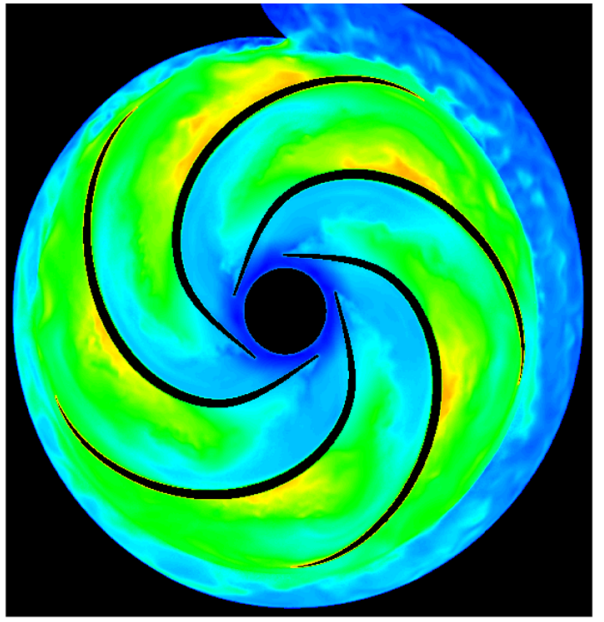

(d)

Figure 8. Variations in the flow velocity (m/s) within the pump for lower head configuration at (a) $6 \mathrm{~m}^{3} / \mathrm{h},(\mathbf{b}) 9 \mathrm{~m}^{3} / \mathrm{h},(\mathbf{c}) 12 \mathrm{~m}^{3} / \mathrm{h}$ and (d) $15 \mathrm{~m}^{3} / \mathrm{h}$. 


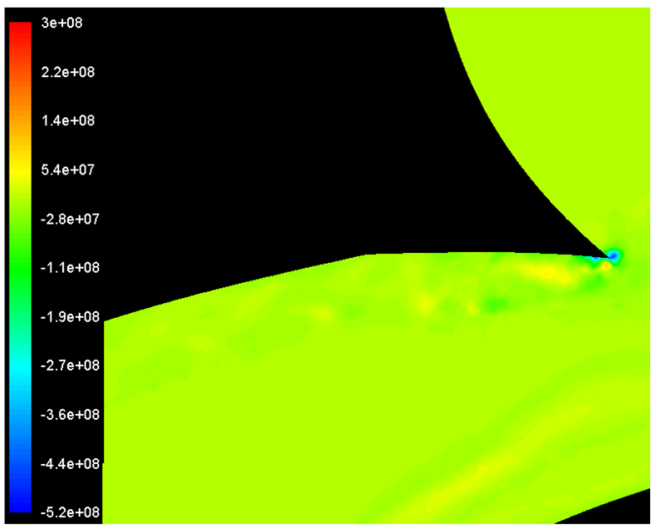

(a)

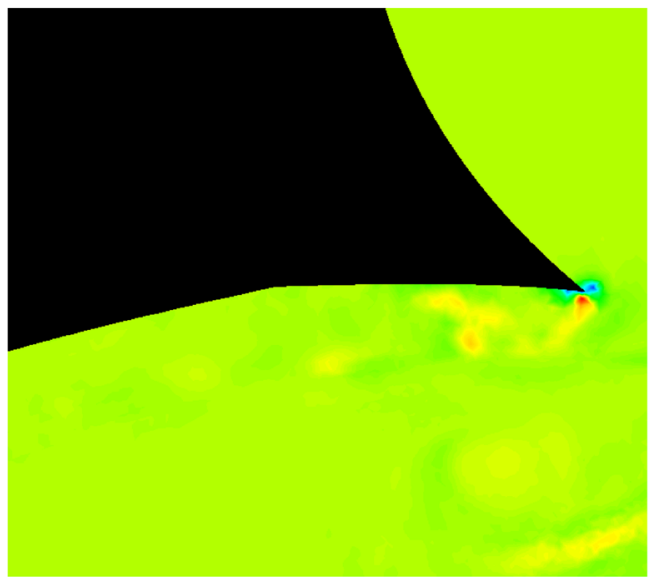

(c)

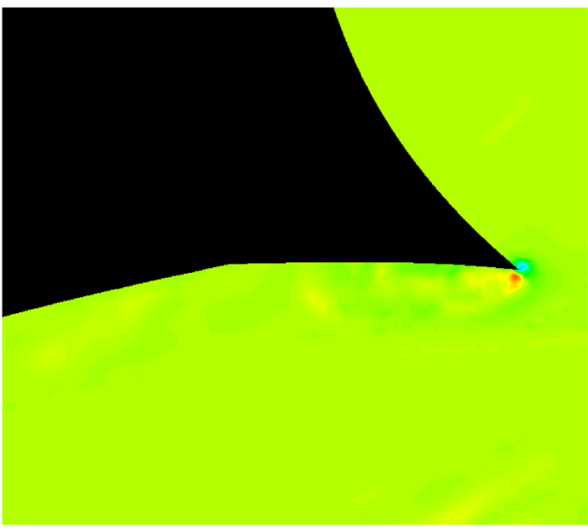

(b)

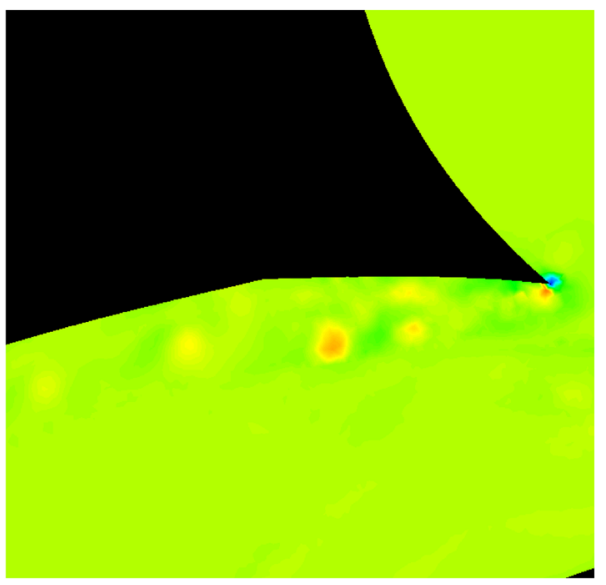

(d)

Figure 9. Variations in $Q$-criterion $\left(/ \mathrm{s}^{2}\right)$ downstream of the tongue region for lower head configuration at (a) $6 \mathrm{~m}^{3} / \mathrm{h},(\mathbf{b}) 9 \mathrm{~m}^{3} / \mathrm{h}$, (c) $12 \mathrm{~m}^{3} / \mathrm{h}$ and (d) $15 \mathrm{~m}^{3} / \mathrm{h}$.

opening available in the near-tongue region, as compared with higher head configuration, where this area is partially blocked by the presence of an impeller blade. Hence, more flow mixing in the near-tongue region is expected (and hence more losses within the volute of the pump) in case of lower head configuration.

In order to ascertain that there are more losses in the near-tongue region for lower head configuration of the pump, $Q$-criteria for lower head configuration have been depicted in figure $9 \mathrm{a}-\mathrm{d}$. The first indication of possibly higher losses comes from the fact that the scale of variations in figure 9 ranges between $-5.2 \times 10^{8}$ and $3 \times 10^{8} / \mathrm{s}^{2}$, whereas the range of the scale in figure 5 is $-4 \times 10^{8}$ to $2.7 \times 10^{8} / \mathrm{s}^{2}$. Hence, the strength of the vortical structures in case of lower head configuration of the pump is $3 \times 10^{7} / \mathrm{s}^{2}$ more as compared with higher head configuration. Furthermore, comparing figures $9 \mathrm{~d}$ and $5 \mathrm{~d}$ reveals that distinct vortical structures (like vortex rings) are shed downstream of the tongue region in case of

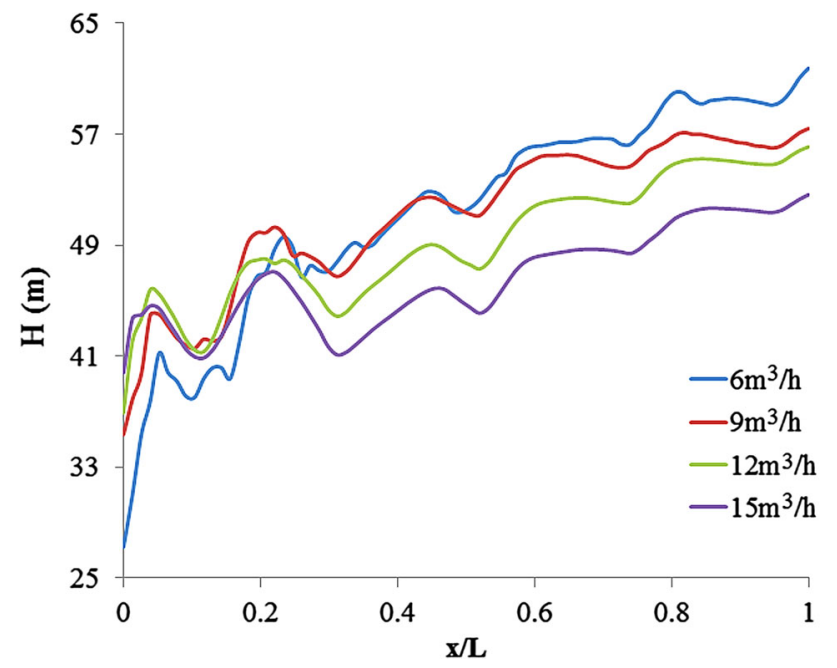

Figure 10. Variations in head developed within the volute for higher head configuration. 


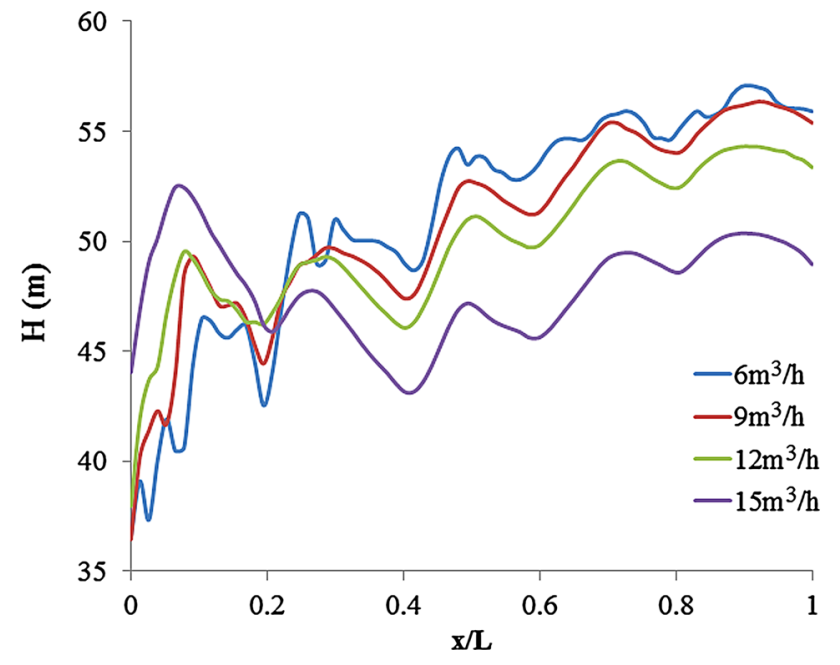

Figure 11. Variations in head developed within the volute for lower head configuration. lower head configuration. Vortical structures are present in case of higher head configuration as well; however, they are more scattered and weak in strength. Hence, these vortical structures are the primary cause for the loss of instantaneous head developed by the pump, and the reason for the formation of these structures is the blade-tongue interaction.

In order to further decipher the instantaneous head developed within the volute of the centrifugal pump, at various operating conditions, figures 10 and 11 have been used to depict the spatial variations of the head at both the higher and lower head configurations. The circumferential distances within the volute (anticlockwise) are shown here on a linear scale, where $x / L$ of 0 and 1 corresponds to the near-tongue region and exit plane of the volute, respectively. It can be clearly seen that as the flow rate increases, the head developed in the volute decreases. Furthermore, the head developed in the volute increases as the

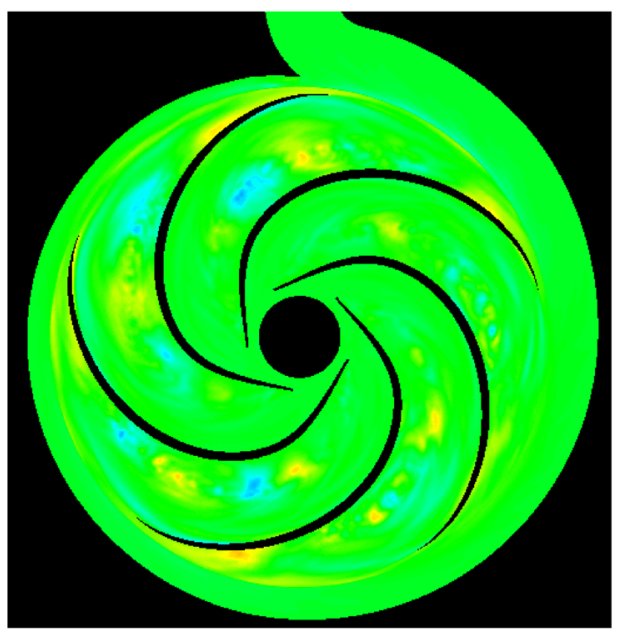

(b)

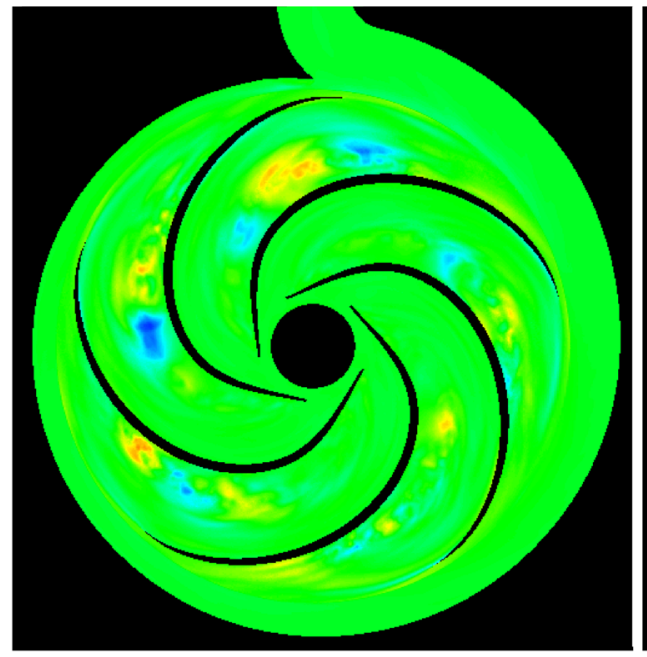

(c)

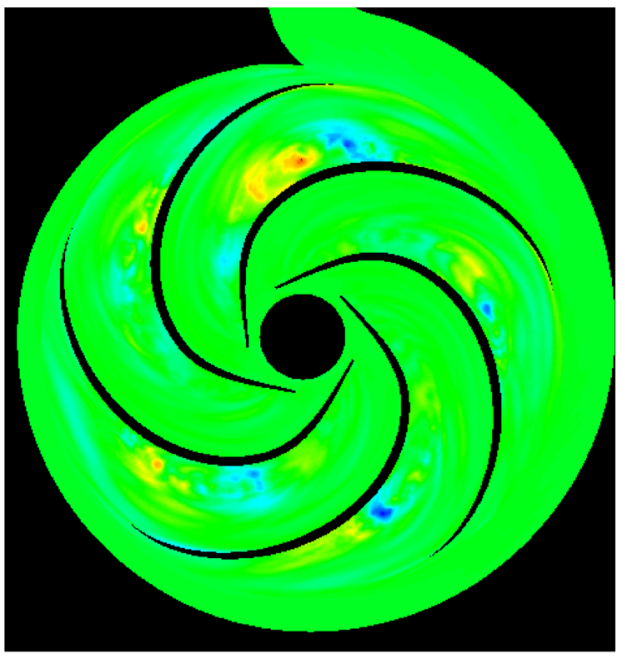

(d)

Figure 12. Variations in resolved $u v$ Reynolds stress $\left(\mathrm{m}^{2} / \mathrm{s}^{2}\right)$ for one revolution of the pump at (a) $6 \mathrm{~m}^{3} / \mathrm{h},(\mathbf{b}) 9 \mathrm{~m}^{3} / \mathrm{h},(\mathbf{c}) 12 \mathrm{~m} / \mathrm{h}$ and (d) $15 \mathrm{~m}^{3} / \mathrm{h}$. 
circumferential distance from the near-tongue region of the volute increases, until the maximum head is obtained at the exit plane of the volute. This trend is the same in both higher and lower head configurations, with two main differences, first being the scale of the variations.

The second notable difference is the fact that the head developed just downstream of the tongue region is higher for higher flow rates, which is contrary to the trend in the rest of the volute. This is due to the presence of vortical structures downstream of the tongue region, which are represented as non-uniform variations in the head curve, where these variations are more abrupt at lower flow rates, and almost non-existent in case of higher flow rates. Hence, lower flow rates exhibit higher losses just downstream of the tongue region, as compared with higher flow rates.

LES provides an opportunity to understand the turbulence behaviour within the flow field. For this purpose, time average resolved $u v$ Reynolds stresses have been computed at different operating conditions and shown in figure 12a-d. Time averaging has been carried out for one complete revolution of the pump as suggested by Rice [21]. Figure 12a shows complex flow features near the blade tips, both on pressure and suction sides, indicating the likelihood of generation of blade tip vortices at lower flow rates. The magnitudes of $u v$ Reynolds stresses change significantly along the circumference near the blade tips, indicating the presence of vortices. This effect seems to become less significant at higher flow rates (figure $12 \mathrm{~b}-\mathrm{d}$ ).

\section{Conclusions}

Transient analysis of a centrifugal pump has been carried out in the present study using LES and Sliding Mesh approaches. The centrifugal pump's performance has been analysed at various operating conditions. It has been shown that LES predicts the complex flow features within a centrifugal pump with reasonable accuracy. Spatio-temporal instantaneous head variations within the centrifugal pump have been analysed critically, along with the flow velocity variations, with the focus on understanding the flow behaviour in the near-tongue region of the pump. It has been concluded that the interaction of impeller blades with the tongue leads to complex secondary flow features called vortical structures. The generation and subsequent dissipation of these vortical structures are strongly dependent on the geometrical orientation of the impeller blades with respect to the tongue. These flow structures are carried downstream of the tongue, and are the primary reason for the head losses within the pump. The higher head points on the head curve correspond to the orientation where the tongue is in-line with an impeller blade, while the lower head points correspond to the tongue in-between two impeller blades. Spatial variations in the head developed by the pump have shown that higher head is developed at higher flow rate just downstream of the tongue region, as these vortical structures lose their strength as the flow rate increases.

\section{References}

[1] Palmer E, Mishra R and Fieldhouse J 2009 An optimisation study of a multiple-row pin-vented brake disc to promote brake cooling using computational fluid dynamics. Proc. Inst. Mech. Eng. Part D 223(7): 865-875

[2] Mishra R, Singh S N and Seshedri V 1998 Study of wear characteristics and solid distribution in constant area and erosion-resistant long-radius pipe bends for the flow of multisized particulate slurries. Wear 217(2): 297-306

[3] Tesfa B, Gu F, Mishra R and Ball A 2014 Emission characteristics of a CI engine running with a range of biodiesel feedstocks. Energies 7(1): 334-350

[4] Hussein M A M and Hasan W K 2013 The effect of rotational speed variation on the velocity vectors in the single blade passage centrifugal pump: part 2. IOSR J. Mech. Civil Eng. 9(2): 43-52

[5] Liu H, Wu X and Tan M 2013 Numerical investigation of the inner flow in a centrifugal pump at the shut-off condition. $J$. Theor. Appl. Mech. 51(3): 649-660

[6] Li D Y, Han L, Wang H J, Gong R Z, Wei X Z and Qin D Q 2016 Pressure fluctuation prediction in pump mode using large eddy simulation and unsteady Reynolds-averaged NavierStokes in a pump-turbine. Adv. Mech. Eng. 8(6): 1-12

[7] Li D Y, Han L, Wang H J, Gong R Z, Wei X Z and Qin D Q 2016 Flow characteristics prediction in pump mode of a pump turbine using large eddy simulation. IMechE Part E: J. Process Mech. Eng. 0(0): 1-17

[8] Ni D, Yang M, Gao B, Zhang N and Li Z 2016 Flow unsteadiness and pressure pulsations in a nuclear reactor coolant pump. J. Mech. Eng. 62(4): 231-242

[9] Zhang N, Yang M, Gao B, Li Z and Ni D 2016 Investigation of rotor-stator interaction and flow unsteadiness in a low specific speed centrifugal pump. J. Mech. Eng. 62(2016): 21-31

[10] Magagnato F and Zhang J 2015 Simulation of a centrifugal pump by using the harmonic balance method. Int. J. Rotating Mach. doi: $10.1155 / 2015 / 729140$

[11] Yao Z F, Yang Z J and Wang F J 2016 Evaluation of nearwall solution approaches for large-eddy simulations of flow in a centrifugal pump impeller. Eng. Appl. Comput. Fluid Mech. 10(1): 454-467

[12] Wang W J, Cui Y R, Wang Y, Li G D, Liang Q H and Yin G 2013 Analysis on the blade inlet pressure fluctuation of the centrifugal pump based on LES. In: Proceedings of the 6th International Conference on Pumps and Fans with Compressors and Wind Turbines, 19-22 September 2013, Beijing, China

[13] Liu H, Dai H, Ding J, Tan M, Wang Y and Huang H 2016 Numerical and experimental studies of hydraulic noise induced by surface dipole sources in a centrifugal pump. $J$. Hydrodyn. 28(1): 43-51

[14] Pump's dimensions obtained from http://www.pedrollo pumps.com/f32200hpage.html

[15] Munson B R, Young D F and Okiishi T H 2002 Fundamentals of fluid mechanics, 4th ed. Wiley, USA 
[16] Ansys 13.0.0 User Guide accessible at http://www1.ansys. com/customer/content/documentation/130/wb2_help.pdf

[17] Smagorinsky J 1963 General circulation experiments with the primitive equations. Mon. Weather Rev. 91(3): 99-164

[18] Patankar S V 1980 Numerical heat transfer and fluid flow. Taylor \& Francis. ISBN 978-0-89116-522-4
[19] Kolar V 2007 Vortex identification: new requirements and limitations. Int. J. Heat Fluid Flow 28(4): 638-652

[20] Haller G 2005 An objective definition of a vortex. J. Fluid Mech. 525: 1-26

[21] Rice M J 2011 High resolution simulation of laminar and transitional flows in a mixing vessel. Ph.D. Thesis, Virginia Tech., USA 